\title{
Interaktionen kardialer und antiretroviraler Medikation
}

\author{
Sabin Egger, Jürgen Drewe ${ }^{1}$
}

\begin{abstract}
Zusammenfassung
Neue therapeutische Optionen der hochaktiven antiretroviralen Therapie (HAART) haben die Morbidität und Mortalität von HIV-infizierten Patienten deutlich gesenkt, so dass nun vermehrt die altersspezifischen Erkrankungen manifest werden, wie z.B. kardiovaskuläre Erkrankungen. Zusätzlich verursacht die HIV-Erkrankung selber eine gewisse
\end{abstract}

\section{Interactions of Cardiac and Antiretroviral Medication}

\begin{abstract}
New therapeutic options such as the highly active antiretroviral therapy (HAART) have significantly improved morbidity and mortality of HIV-infected patients. Consequently, age-related diseases become more manifest. In addition, HIV infection it-
\end{abstract}

${ }^{1}$ Abteilung für Klinische Pharmakologie \& Toxikologie, Universitätsspital Basel, Schweiz. kardiovaskuläre Morbidität. Aus diesem Grund gewinnt die Behandlung der kardiovaskulären Symptome eine zunehmende Bedeutung. Die Pharmakotherapie dieser HIV-positiven Patienten ist sehr komplex, da sie durch eine Polypharmazie aus Medikamenten mit einem hohen Interaktionspotential geprägt ist.

\section{Schlüsselwörter:}

Interaktionen · Cytochrom $\cdot$ Antiretrovirale Therapie $\cdot$ Kardiovaskuläre Medikamente

Herz 2005;30:493-503

DOI $10.1007 /$

s00059-005-2723-4

self causes certain cardiovascular morbidity. Therefore, treatment of cardiovascular symptoms of HIV-positive patients becomes more and more important. Pharmacotherapy in these patients is complex and requires polypharmacy with drugs carrying a high risk of drug-drug interactions.

\section{Key Words:}

Drug interactions - Cytochrome $\cdot$ Antiretroviral therapy · Cardiovascular drugs

\section{Einleitung}

Mit der Einführung der neuen hochaktiven antiretroviralen Therapie (HAART) konnten die HIV-Morbidität und -Mortalität erheblich vermindert werden. HIV-infizierte Patienten werden nun älter und zeigen vermehrt die altersentsprechenden kardiovaskulären Erkrankungen wie in der Kontrollgruppe ohne HIV-Infektion.

$\mathrm{Zu}$ dieser Morbidität kommen nun auch spezifische kardiovaskuläre Erkrankungen hinzu, die durch die Virusinfektion direkt oder indirekt verursacht worden sind [1], wie z.B.

1. durch das HIV oder Begleitinfektionen oder Drogen (z.B. Kokain) verursachte Myokarditis und nachfolgende Kardiomyopathie,

2. erhöhte Prävalenz von (infektiösen) Perikarditiden und Endokarditiden (durch HIV, Begleitinfektionen oder durch Malignome, Malnutrition),

3. rechtsventrikuläre und pulmonale Erkrankungen; pulmonalarterielle Hypertonie (Prävalenz bei HIV deutlich erhöht),

4. Vaskulitis (als unerwünschte Arzneimittelwirkungen [UAWs] der antiinfektiösen und antiretroviralen Therapie),
5. beschleunigte Arteriosklerose ( $8 \%$ der Patienten mit HIV-Protease-Inhibitoren),

6. Arrhythmien (UAWs, autonome Dysfunktion),

7. Lipodystrophie (HIV-Protease-Inhibitoren).

\section{Typen von Interaktionen}

Der kardiovaskulär erkrankte HIV-positive Patient stellt eine große Herausforderung für die behandelnden Ärzte dar: Er benötigt in der Regel eine komplexe Polypharmazie. Diese besteht z.T. aus Medikamenten mit einem hohen Interaktionspotential. Die daraus folgenden Interaktionen können pharmakokinetischer und/oder pharmakodynamischer Natur sein. Bei den pharmakokinetischen Interaktionen beeinflusst ein Medikament die Aufnahme (Absorption), den Abbau (Metabolismus) oder die Ausscheidung eines oder mehrerer anderer gleichzeitig gegebener Medikamente. Bei den pharmakodynamischen Interaktionen beeinträchtigt ein Medikament die Effekte eines oder mehrerer anderer Medikamente. 
Die häufigste Ursache einer pharmakokinetischen Interaktion ist auf der Ebene des Metabolismus zu finden. Ziel des Metabolismus ist es, die in der Regel lipophilen und dadurch vom Körper schwer ausscheidbaren Medikamente durch chemische Modifikation (Phase-I-Reaktionen) oder durch Konjugation mit wasserlöslichen Säuren wie z.B. Glucuronsäure (Phase-II-Reaktionen) in wasserlöslichere Formen umzuwandeln, die der Körper leichter ausscheiden kann. Die Phase-I-Reaktionen werden zum größten Teil durch die Enzyme des Cytochrom-P450-Enzymsystems vermittelt. Diese Enzyme sind sehr empfindlich für Interaktionen. Die wichtigsten Isoenzyme (CYPs) für den Abbau von Medikamenten sind das CYP 3A4, 2D6, 2C $9 / 2 \mathrm{C} 19$ und $1 \mathrm{~A} 2$, welche ungefähr $50 \%, 25 \%$, $20 \%, 5 \%$ und $1 \%$ der heutigen Medikamente metabolisieren.

\section{Welche Interaktionen sind denkbar?}

Werden die CYPs gehemmt, so hat dies eine Erhöhung der Blutspiegel der von ihnen abgebauten Medikamente zur Folge, was zu einem verstärkten Effekt oder einer dosisabhängigen Toxizität führen kann. Genetische Mutationen in den für die Enzyme kodierenden Genen können ebenfalls zu einer (permanent) verminderten Menge an funktionstüchtigem Enzym und zum Phänotyp eines „langsamen Metabolisierers" führen. Solche Mutationen werden, wenn sie in einer Population eine bestimmte Häufigkeit aufweisen (> 1\%), Polymorphismen genannt. Sie sind für die CYPs 2D6, 2C19 und 2C9 beschrieben und treten in Westeuropa mit einer Prävalenz von $10 \%, 2 \%$ und $1 \%$ auf. Des Weiteren kann es zu Genduplikationen kommen, so dass das entsprechende Gen mehrfach vorkommt und permanent verstärkt exprimiert wird. Dies führt dann zu einer permanenten Zunahme der aktiven Enzymmenge, einem er-

Tabelle 1. Typen der metabolischen Interaktionen.

Table 1. Types of metabolic interactions.

\begin{tabular}{|c|c|c|c|}
\hline Typ & $\begin{array}{l}\text { Meta- } \\
\text { bolismus }\end{array}$ & $\begin{array}{l}\text { Blut- } \\
\text { spiegel }\end{array}$ & Effekte \\
\hline Enzymhemmung & Vermindert & Erhöht & Erhöht/Toxizität \\
\hline $\begin{array}{l}\text { Polymorphismus } \\
\text { („„langsamer Metabolisierer“) }\end{array}$ & Vermindert & Erhöht & Erhöht/Toxizität \\
\hline $\begin{array}{l}\text { Polymorphismus } \\
\text { („ultraschneller Metabolisierer“) }\end{array}$ & Erhöht & Erniedrigt & Erniedrigt/Wirkungsverlust \\
\hline Enzyminduktion & Erhöht & Erniedrigt & Erniedrigt/Wirkungsverlust \\
\hline
\end{tabular}

höhten Metabolismus und verminderten Blutspiegeln der von dem Enzym abgebauten Medikamente. In diesen Fällen sind die Effekte des jeweiligen entsprechenden Medikaments bei normalerweise wirksamen Dosierungen vermindert bis hin zum totalen Wirkungsverlust. Der resultierende Phänotyp wird auch „ultraschneller Metabolisierer"genannt. Ähnliche Auswirkungen hat eine Enzyminduktion des jeweiligen Cytochroms: hier liegt eine in diesem Fall temporär erhöhte Menge des aktiven Enzyms vor (s. Tabelle 1).

Die verschiedenen Typen der Interaktionen unterscheiden sich erheblich durch den Zeitverlauf ihres Eintretens und ihrer Dauer: Eine Enzymhemmung setzt sehr schnell nach Gabe des Hemmstoffs ein und dauert nach Absetzen entsprechend der Halbwertszeit des Hemmstoffs im Blut Stunden bis Tage. Eine Enzyminduktion setzt erst 2-3 Tage nach Gabe des Induktors ein und kann sogar erst nach 1-2 Wochen ihren Höhepunkt erreichen (z.B. Carbamazepin). Die Wirkung der Enzyminduktion hält nach Absetzen des Induktors je nach Halbwertszeit der Substanz und des induzierten Enzyms einige Tage bis Wochen länger an.

Eine wichtige Rolle bei der Aufnahme von Medikamenten aus dem Darm, der Verteilung im Körper (z.B. Blut-Hirn-Schranke) und bei der renalen und hepatischen Elimination von Medikamenten spielen Transportproteine. Das wichtigste dieser Transportproteine ist das P-Glykoprotein (Pgp). Es besitzt eine breite Substratspezifität und transportiert eine Vielzahl strukturell unterschiedlicher Medikamente.

Im Folgenden werden die wichtigsten antiretroviralen und kardiovaskulären Medikamente hinsichtlich ihres Abbaus und der möglichen Interaktionen besprochen.

\section{Wichtige HIV-Medikamente HIV-Protease-Inhibitoren}

Die Wirkungen der HIV-Protease-Inhibitoren Saquinavir, Indinavir, Ritonavir, Nelfinavir, Amprenavir, Lopinavir, Fosamprenavir (Prodrug von Amprenavir), Atazanavir auf CYPs, Pgp und UDP-Glucuronosyltransferase (UGT) sind in Tabellen 2a bis 2c dargestellt.

Ritonavir inhibiert sehr stark den Metabolismus von CYP3A4-Substraten in vitro [2]. Diese Inhibition konnte ebenfalls in verschiedenen klinischen Studien [2] nach Kurzzeitverabreichung von Ritonavir gezeigt werden. Nach Langzeitverabreichung scheint Ritonavir hingegen CYP3A4 zu induzieren [2]. 
Nichtnukleosidische Reverse-

Transkriptase-Inhibitoren (NNRTIs)

Die Interaktionen von Nevirapin, Delavirdin und Efavirenz sind in Tabellen 3a bis 3c dargestellt.

\section{Nukleosid- und Nukleotid-Reverse- Transkriptase-Inhibitoren (NRTIs)}

Die NRTIs Zidovudin, Didanosin, Zalcitabin, Stavudin, Lamivudin, Abacavir, Tenofovir Disoproxil, Adefovir Dipivoxil und Emtricitabin werden nicht über CYPs metabolisiert. Didanosin, Zalcitabin, Stavudin, Lamivudin und Adefovir werden renal ausgeschieden. Abacavir wird glucuronidiert und ist ein Substrat der Alkoholdehydrogenase. Es sind keine relevanten Interaktionen zu erwarten [3].

\section{Fusionsinhibitoren}

Enfuvirtid ist ein synthetisches Peptid, das am HIV-1-Glykoprotein 41 bindet und die Fusion der Virusmembran mit der Zellmembran blockiert [4]. Enfuvirtid wird durch Proteolyse zu Aminosäureresten abgebaut. Aus diesem Grund ist das Interaktionspotential gering [4]. Es beeinflusst den $\mathrm{Me}$ tabolismus anderer Medikamente, welche durch CYPs abgebaut werden, nicht [5]. Daher haben Enzyminhibitoren wie Ritonavir oder Saquinavir oder Enzyminduktoren wie Rifampicin keinen Effekt auf die Pharmakokinetik von Enfuvirtid [4].

\section{Antibiotika und Antimykotika}

HIV-positive Patienten stehen häufig unter zusätzlicher Therapie mit Antibiotika, Antiviralia und/oder Antimykotika.

\section{Antiviralia: Nukleosid- and Nukleotid-Analoga}

Die Nukleosid- und Nukleotid-Analoga Aciclovir, Ganciclovir, Famciclovir, Valaciclovir, Penciclovir, Valganciclovir, Ribavirin und Cidofovir werden nicht durch CYPs abgebaut und interagieren nicht damit. Somit ist das Interaktionspotential mit kardiovaskulären Medikamenten gering.

\section{Trimethoprim-Sulfamethoxazol (Co-trimoxazol)}

Zur Pneumocystis-carinii-Prophylaxe wird häufig Trimethoprim-Sulfamethoxazol verwendet. Sulfamethoxazol wird über $\mathrm{CYP} 2 \mathrm{C} 9$ abgebaut und hemmt dieses Enzym. Trimethoprim ist ein schwa-
Tabelle 2a. CYP-Substrate - HIV-Protease-Inhibitoren. Pgp: P-Glykoprotein; UGT: UDP-Glucuronosyltransferase.

Table 2a. Substrates for CYP enzymes - HIV protease inhibitors. Pgp: P-glycoprotein; UGT: UDP-glucuronosyltransferase.

\begin{tabular}{|c|c|c|c|c|c|c|c|c|}
\hline & $1 \mathrm{~A} 2$ & $2 \mathrm{~B} 6$ & $2 \mathrm{CO}$ & $2 \mathrm{C} 19$ & 2D6 & $3 \mathrm{~A} 4$ & Pgp & UGT \\
\hline Saquinavir $[89,90]$ & & & & & & $x$ & $x$ & \\
\hline Indinavir [91] & & & & & & $x$ & $x$ & \\
\hline Ritonavir $[92,93]$ & $(x)$ & $(x)$ & & & $x$ & $x$ & & UGT1A1 \\
\hline Nelfinavir [94] & & & & $x$ & & $x$ & & \\
\hline Amprenavir [95] & & & $(\mathrm{x})$ & & $(x)$ & $x$ & & \\
\hline Lopinavir $[92,96]$ & & & & & & $x$ & & \\
\hline Fosamprenavir [95] & & & $(\mathrm{x})$ & & $(x)$ & $x$ & & \\
\hline Atazanavir [97] & & & & & & $x$ & $x$ & \\
\hline
\end{tabular}

Tabelle 2b. CYP-Inhibitoren - HIV-Protease-Inhibitoren. Abkürzungen s. Tabelle 2 a.

Table $\mathbf{2 b}$. Inhibitors of CYP enzymes - HIV protease inhibitors. Abbreviations see Table $2 a$.

\begin{tabular}{|c|c|c|c|c|c|c|c|c|c|}
\hline & $1 \mathrm{~A} 2$ & 2B6 & $2 \mathrm{CO}$ & $2 C 19$ & 2D6 & $2 \mathrm{E} 1$ & $3 \mathrm{~A} 4$ & Pgp & UGT \\
\hline Saquinavir [94, 97-99] & & & + & & + & & ++ & ++ & \\
\hline Indinavir $[94,98]$ & & & & & + & & +++ & & \\
\hline $\begin{array}{l}\text { Ritonavir initial } \\
{[94,97,100-102]}\end{array}$ & & ++ & ++ & + & ++ & $(++)$ & +++ & + & \\
\hline \multicolumn{10}{|l|}{ Ritonavir chronisch } \\
\hline Nelfinavir $[94,98,100,103]$ & & ++ & & & + & & ++ & & \\
\hline Amprenavir akut [94] & & & & $(++)$ & & & +++ & & \\
\hline Lopinavir akut $[91,104]$ & & & & & & & + & ++ & \\
\hline
\end{tabular}

Tabelle 2c. CYP-Induktoren - HIV-Protease-Inhibitoren. Abkürzungen s. Tabelle 2 a. Table 2c. Inducers of CYP enzymes - HIV protease inhibitors. Abbreviations see Table $2 a$.

1A2 2B6 2C9 2C19 2D6 2E1 3A4 Pgp UGT

Saquinavir

Indinavir

Ritonavir initial

UGT1A1

$\begin{array}{lllllll}\text { Ritonavir chronisch } & \mathrm{x} & \mathrm{x} & \mathrm{x} & \mathrm{x} & \mathrm{x} & \text { UGT1A1 }\end{array}$

$[91,92,105,106]$

Nelfinavir [107]

Amprenavir chronisch [107]

Lopinavir chronisch [104]

Fosamprenavir

Atazanavir [108].

\section{$x \quad x$}

$x \quad x$

$x$

$x$

cher Inhibitor von CYP2C9 [6]. Interaktionen mit

Warfarin sind in der Literatur beschrieben.

\section{Rifampicin and Rifabutin}

Rifabutin [7] und Rifampicin [8] werden hauptsächlich durch Esterasen metabolisiert. Rifampi- 
Tabelle за. CYP-Substrate - nichtnukleosidische Reverse-Transkriptase-Inhibitoren. Abkürzungen s. Tabelle $2 a$.

Table зa. Substrates for CYP enzymes - non-nucleoside reverse transcriptase inhibitors. Abbreviations see Table 2 a.

\begin{tabular}{|c|c|c|c|c|c|c|}
\hline & $1 \mathrm{~A} 2$ & $2 \mathrm{~B} 6$ & $2 \mathrm{CO}$ & 2D6 & $3 \mathrm{~A} 4$ & Pgp UGT \\
\hline Nevirapin $[109,110]$ & & $x$ & & & $x$ & \\
\hline Delavirdin [111] & & & & $x$ & $x$ & \\
\hline Efavirenz $[109,112]$ & x (Nebenweg) & $x$ (Hauptweg) & & & x (Nebenweg) & \\
\hline
\end{tabular}

Tabelle 3b. CYP-Inhibitoren - nichtnukleosidische Reverse-Transkriptase-Inhibitoren. Abkürzung s. Tabelle 2a.

Table $\mathbf{3}$ b. Inhibitors of CYP enzymes - non-nucleoside reverse transcriptase inhibitors. Abbreviation see Table 2 a.

\begin{tabular}{|c|c|c|c|c|c|c|}
\hline & $1 \mathrm{~A} 2$ & 2B6 & $2 \mathrm{C} 9$ & $2 C 19$ & 2D6 & 3A4 Pgp \\
\hline Nevirapin [113] & $\begin{array}{l}<+ \\
\text { (vernachlässigbar } \\
\text { in vitro) }\end{array}$ & & & & $\begin{array}{l}<+ \\
\text { (vernachlässigbar } \\
\text { in vitro) }\end{array}$ & + \\
\hline Delavirdin $[111,113]$ & $\begin{array}{l}<+ \\
\text { (vernachlässigbar } \\
\text { in vitro) }\end{array}$ & & ++ & + & $\begin{array}{l}<+ \\
\text { (vernachlässigbar } \\
\text { in vitro) }\end{array}$ & +++ \\
\hline Efavirenz [113] & $\begin{array}{l}<+ \\
\text { (vernachlässigbar } \\
\text { in vitro) }\end{array}$ & & ++ & ++ & $\begin{array}{l}<+ \\
\text { (vernachlässigbar } \\
\text { in vitro) }\end{array}$ & ++ \\
\hline
\end{tabular}

Tabelle зc. CYP-Induktoren - nichtnukleosidische Reverse-Transkriptase-Inhibitoren. Abkürzungen s. Tabelle $2 a$.

Table 3c. Inducers of CYP enzymes - non-nucleoside reverse transcriptase inhibitors. Abbreviations see Table $2 a$.

\begin{tabular}{|c|c|c|c|c|c|c|c|c|c|}
\hline & $1 \mathrm{~A} 2$ & 2B6 & $2 C 9$ & 2C19 & 2D6 & 2E1 & $3 A 4$ & Pgp & UGT \\
\hline Nevirapin $[109,110,114]$ & & $x$ & & & & & $x$ & $x$ & \\
\hline Efavirenz $[109,115]$ & & & & & & & $x$ & & \\
\hline
\end{tabular}

cin ist jedoch ein starker Induktor vieler Cytochrome (1A2, 2A6, 2B6, 2C8, 2C9, 2C19, 3A4 [!!]) sowie von Pgp und UGT [9]. Rifabutin hingegen ist nur ein schwacher Induktor von CYP3A4 [10].

Eine volle Induktion wird etwa 1 Woche nach Start der Rifampicin-Behandlung beobachtet und ist etwa 2 Wochen nach Absetzen von Rifampicin vollständig verschwunden [9].

Rifampicin hat den größten Effekt auf die Pharmakokinetik bei Medikamenten mit einem extensiven First-Pass-Metabolismus [9]: Es führt zu klinisch relevanten Interaktionen mit den folgenden Medikamenten (Serumspiegel meist vermindert):

- antiretrovirale Medikamente;

- Antimykotika;

- Co-trimoxazol;

- Antiarrhythmika: z.B. Amiodaron;
- Digoxin (wegen Pgp-Induktion);

- Sildenafil: Serumspiegel werden erwartungsgemäß vermindert. Dies ist relevant, wenn Sildenafil zur Behandlung der pulmonalen Hypertonie eingesetzt wird (Wirkungsverlust).

- HMG-CoA-Reductase-Inhibitoren.

Interaktionen mit HIV-Protease-Inhibitoren oder NRTIs. Rifampicin ist ein starker Induktor verschiedener CYPs und vermag die Konzentration verschiedener antiretroviraler Medikamente zu senken. Richtlinien für die Prophylaxe und Behandlung einer Tuberkulose bei HIV-positiven Patienten erlauben die Verwendung von Rifampicin oder Rifabutin nur bei den Patienten mit einer der folgenden Behandlungen:

- NNRTI Efavirenz und zwei NRTIs oder

- Ritonavir und ein oder mehrere NRTIs oder

- Kombination von zwei HIV-Protease-Inhibitoren (Ritonavir oder Saquinavir).

Bei Patienten mit Ritonavir sollte die Dosis von Rifabutin vermindert und bei Patienten mit Efavirenz erhöht werden [11]. Da die NRTIs nicht über CYPs metabolisiert werden, erwartet man keine Interaktion.

Interaktion zwischen Rifampicin/Rifabutin und Azol-Antimykotika. Plasmakonzentrationen von Ketoconazol, Fluconazol und Itraconazol werden deutlich vermindert [9].

\section{Isoniazid (INH)}

Das Tuberkulostatikum Isoniazid wird vor allem über die N-Acetyltransferase 2 (NAT2) abgebaut [12]. Es existieren Polymorphismen der NAT2 (Prävalenz ca. 50\%), was zu einer großen Variabilität in den Plasmakonzentrationen führt. INH inhibiert verschiedene CYPs (1A2, 2A6, 2C9, 2C19, 2E1 und 3A4) [13] und induziert CYP2E1 [14].

Interaktion zwischen Rifampicin und INH. Koadministration von INH und Rifampicin erhöht möglicherweise die Hepatotoxizität [15] (von $1,6 \%$ bei INH-Monotherapie auf $2,5-2,7 \%$ bei Kombinationstherapie [16]). Es wird vermutet, dass Rifampicin den Abbau von INH zu toxischen Metaboliten fördert.

\section{Pyrazinamid}

Das Tuberkulostatikum Pyrazinamid wird nicht durch Cytochrome metabolisiert [17] und zeigt keine inhibitorischen Effekte auf Cytochrome [13]. Aus diesem Grund sind keine pharmakokinetischen Interaktionen zu erwarten. Die Hepatotoxizität von Pyrazinamid kann aber durch gleichzeitige Gabe von INH oder Rifampicin erhöht werden [18]. 


\section{Ethambutol}

Das Tuberkulostatikum Ethambutol wird hauptsächlich renal eliminiert [19]. Nach oraler Gabe werden 50-70\% der Dosis unverändert renal ausgeschieden. Der Metabolismus geschieht in der Leber über die Alkohol- und Aldehyddehydrogenase. Es sind keine pharmakokinetischen Interaktionen zu erwarten.

\section{Azol-Antimykotika}

Die Interaktion der Azol-Antimykotika Fluconazol, Voriconazol, Itraconazol, Miconazol und Ketoconazol ist in Tabellen $4 \mathrm{a}$ und $4 \mathrm{~b}$ dargestellt.

Ketoconazol ist ein potenter Inhibitor des CYP3A4 [20]. Fluconazol ist dagegen ein relativ schwacher Inhibitor. Dosen $\geq 200 \mathrm{mg}$ pro Tag reichen aus, den Metabolismus von CYP3A4-Substraten relevant $\mathrm{zu}$ hemmen.

Interaktionen wurden vor allem bei Koadministration mit Rifampicin beobachtet [14].

\section{Wichtige kardiovaskuläre \\ Medikamente \\ Orale Antikoagulanzien}

Die oralen Antikoagulanzien Acenocoumarol, Phenprocoumon und Warfarin werden über verschiedene Cytochrome abgebaut, wie Tabelle 5 zeigt.

Interaktion zwischen Ritonavir/Nelfinavir und Warfarin. Die akute Gabe von Ritonavir führt zu einer Inhibition von CYP2C9, eine chronische Gabe zu einer Induktion. Bei einem Patienten unter Warfarin-Therapie führte die Gabe von Ritonavir nach ein paar Wochen zu einer Verminderung des Warfarin-Effekts, und die Warfarin-Dosis musste erhöht werden. Nach Absetzen von Ritonavir stieg die INR (International Normalized Ratio) an, und die Dosiserhöhung von Warfarin musste wieder rückgängig gemacht werden [21]. Ein ähnlicher induktiver Effekt auf CYP2C9 wurde unter gleichzeitiger Gabe von Nelfinavir beobachtet [22]. Die akute Gabe von Ritonavir bei Warfarin-Therapie erhöhte in einem anderen Fall die INR von 2,4-3,0 auf 10,4, ohne dass Anzeichen einer Blutung beobachtet wurden [23]. Ähnliche Verstärkungen des gerinnungshemmenden Effekts durch akute Ritonavir-Gabe wurden ebenfalls unter Acenocoumarol berichtet [24].

Interaktion zwischen Warfarin und Trimethoprim-Sulfamethoxazol. Eine Wirkungsverstärkung von Warfarin durch Gabe von Trimethoprim-Sulfamethoxazol wurde mehrfach berichtet, welche teilweise $\mathrm{zu}$ Blutungen führte [25-27].
Tabelle 4a. CYP-Substrate - Azol-Antimykotika.

Table 4a. Substrates for CYP enzymes - azole antimycotics.

\begin{tabular}{llll}
\hline & $2 \mathrm{C9}$ & $2 \mathrm{2C19}$ & $3 \mathrm{A4}$ \\
\hline Fluconazol & & \\
Voriconazol [116] & $\times$ (Nebenweg) & $\times$ & $\times$ \\
Itraconazol [117] & & $\times$ \\
Miconazol & & $\mathrm{x}$ \\
Ketoconazol [118] & &
\end{tabular}

Tabelle 4b. CYP-Inhibitoren - Azol-Antimykotika. Abkürzung s. Tabelle 2a.

Table $\mathbf{4 b}$. Inducers of CYP enzymes - azole antimycotics. Abbreviation see Table 2a.

\begin{tabular}{lcccccccc}
\hline & 1A1/2 & 2 A6 & 2C8 & 2C9 & 2 2C19 & 2E1 & 3A4 & Pgp \\
\hline Fluconazol [20] & & & & ++ & ++ & & + & \\
Voriconazol [20] & & & & & & & ++ & \\
Itraconazol [20, 117] & & & & & & & ++ & ++ \\
Miconazol [20] & & ++ & & ++ & & ++ & ++ & \\
Ketoconazol [20] & ++ & + & + & + & + & & +++ & ++ \\
\hline
\end{tabular}

Tabelle 5. CYP-Substrate - orale Antikoagulanzien.

Table 5. Substrates for CYP enzymes - oral anticoagulants.

\begin{tabular}{llllll}
\hline & 1A2 & 2C9 & 2C19 & 2C8 & 3A4 \\
\hline Phenprocoumon [119] & & $x$ & & $x$ & $x$ \\
Acenocoumarol [120] & $\times$ & $\times$ & $x$ & & \\
Warfarin [121, 122] & $\times$ & $\times$ (Hauptweg) & & \\
\hline
\end{tabular}

Diese Interaktion kann zumindest teilweise mit einer Hemmung des CYP2C9-vermittelten Warfarin-Metabolismus durch Sulfamethoxazol erklärt werden.

Interaktion zwischen oralen Antikoagulanzien und Rifampicin/Rifabutin. Rifampicin kann den Metabolismus von Acenocoumarol, Phenprocoumon und Warfarin induzieren [28-30], so dass die Dosierung möglicherweise erhöht werden muss.

Interaktion zwischen oralen Antikoagulanzien und Azol-Antimykotika. Fluconazol, Ketoconazol, Itraconazol und Voriconazol inhibieren den Abbau von oralen Antikoagulanzien und führen zu verstärkten Effekten [20,31-33]. In einer Kasuistik wurde sogar nach topischer Anwendung von Miconazol eine Verstärkung des gerinnungshemmenden Effektes von Acenocoumarol berichtet [34].

Interaktion zwischen oralen Antikoagulanzien und Isoniazid. Isoniazid kann in sehr hohen Dosen (d.h. $600 \mathrm{mg} / \mathrm{d}$ ) den Metabolismus verschiedener Medikamente, so auch von oralen Antikoagulanzien, inhibieren [32]. 
Tabelle 6. CYP-Inhibitor - Amiodaron. Abkürzung s. Tabelle 2a.

Table 6. Inhibitor of CYP enzymes - amiodarone. Abbreviation see Table $2 a$.

\begin{tabular}{|c|c|c|c|c|c|c|c|c|c|}
\hline & $1 \mathrm{~A} 2$ & $2 A 6$ & 2B6 & $2 \mathrm{C} 8$ & $2 \mathrm{C} 9$ & $2 C 19$ & 2D6 & $3 A 4$ & Pgp \\
\hline Amiodaron [45] & & & & $(+)$ & + & & ++ & ++ & $(++)$ \\
\hline N-Desethylamiodaron [45] & + & + & + & $(++)$ & ++ & + & ++ & + & \\
\hline
\end{tabular}

Thrombozytenaggregationshemmer

(Acetylsalicylsäure, Clopidogrel)

Acetylsalicylsäure wird nicht über Cytochrome abgebaut, Clopidogrel wird vor allem über das CYP3A4 abgebaut (aktiviert). Nebenwege des Abbaus führen über CYP1A2 und CYP2B6. Andererseits inhibiert es selbst stark CYP2B6 und weniger stark CYP2C9 und CYP2C19 [35]. Es finden sich nur wenige Angaben zu Interaktionen zu Clopidogrel oder Therapie der HIVInfektion.

CYP3A4 aktiviert Clopidogrel [36]. Aus diesem Grund ist denkbar, dass CYP3A4-Inhibitoren die Thrombozytenaggregation erhöhen, während CYP3A4-Induktoren wie z.B. Rifampicin diese vermindern. Es konnten allerdings keine Fallberichte zu relevanten Interaktionszwischenfällen gefunden werden.

\section{Bosentan}

Der Endothelin-Antagonist Bosentan ist das Mittel der Wahl bei der Therapie der pulmonalarteriellen Hypertonie, die bei HIV-infizierten Patienten häufiger vorkommt. Bosentan wird von CYP2C9 und CYP3A4 abgebaut [37] und induziert ebenfalls diese beiden Enzyme [37].

Es wurden keine Interaktionen zwischen Bosentan und antiretroviralen Medikamenten beschrieben. Andererseits konnten etwa zweifach erhöhte Plasmakonzentrationen von Bosentan bei gleichzeitiger Therapie mit Ketoconazol beobachtet werden [38]. Es ist nicht auszuschließen, dass andere Inhibitoren von CYP3A4 oder CYP2C9 ebenfalls die Plasmakonzentrationen erhöhen können.

\section{Sildenafil}

Sildenafil ist ein Hemmer der Phosphodiesterase Typ 5, der in der Therapie der pulmonalen Hypertonie eingesetzt werden kann. Es ist Substrat des CYP3A4 (Hauptweg) und CYP2C9 (Nebenweg) $[39,40]$.

Interaktion zwischen Indinavir, Saquinavir oder Ritonavir und Sildenafil. Saquinavir, Indinavir und Ritonavir erhöhen die Plasmaspiegel von Sildena- fil durch Hemmung von CYP3A4 (Ritonavir zusätzlich weniger stark CYP2C9) [39, 41]. Aus diesem Grund sollte bei Patienten unter Indinavir, Saquinavir und/oder Ritonavir mit einer niedrigen Anfangsdosis von Sildenafil begonnen werden.

\section{Amiodaron}

Amiodaron ist ein Substrat der Cytochrome 2C8 und 3A4 [42]. Es besitzt einen aktiven Metaboliten: N-Desethylamiodaron. Es wird über Pgp und/oder den organischen Anionentransporter OATP2 transportiert [43, 44]. Amiodaron und sein aktiver Metabolit hemmen selbst verschiedene Cytochrome (s. Tabelle 6)

Interaktion zwischen HIV-Protease-Inhibitoren und Amiodaron. Monitoring der AmiodaronToxizität (Bradykardie, Hypotonie, Verlängerung der QTc-Zeit) wird empfohlen für antiretrovirale Medikamente, die CYP3A4 hemmen, wie Indinavir [46] und andere HIV-ProteaseInhibitoren [47].

Interaktion zwischen Rifampicin/Rifabutin und Amiodaron. Gleichzeitige Gabe von Amiodaron und Rifampicin/Rifabutin kann zu einem Wirkungsverlust von Amiodaron führen [48].

\section{Digoxin}

Digoxin wird nicht über CYP metabolisiert, ist aber ein Substrat des Pgp [49].

Interaktion zwischen Ritonavir und Digoxin. Ritonavir-Gabe führt zu einer Erhöhung der Plasmakonzentrationen von Digoxin. Dieser Effekt erklärt sich vermutlich durch eine Inhibition des Pgp, welche die intestinale Aufnahme von Digoxin erhöht und die renale Elimination vermindert [50-52].

Interaktion zwischen Azol-Antimykotika und Digoxin. Die Interaktionen zwischen Digoxin und Azol-Antimykotika (Erhöhung der Digoxin-Konzentrationen durch Itraconazol, Ketoconazol) können vermutlich ebenfalls durch Hemmung des Pgp erklärt werden [20, 53, 54].

Interaktion zwischen Rifampicin/Rifabutin und Digoxin. Rifampicin/Rifabutin führen zu stark erniedrigten Plasmakonzentrationen von Digoxin [55]. In dieser pharmakokinetischen Studie konnte die gesteigerte intestinale Pgp-Expression (3,5fach erhöht im Vergleich zur Kontrolle) gezeigt werden. Die renale Clearance war in der Studie nicht verändert, so dass die erniedrigten Digoxin-Plasmaspiegel durch eine verminderte enterale Aufnahme wegen einer gesteigerten intestinalen Pgp-Aktivität erklärt werden könnten. 


\section{$\beta$-Blocker}

Die meisten $\beta$-Blocker werden über CYP2D6 metabolisiert [3, 56]. Nadolol, Sotalol, Atenolol (hydrophile $\beta$-Blocker) werden hauptsächlich renal eliminiert [56]. Spezialfälle sind Propranolol, Carvedilol und Bisoprolol, die über mehrere CYPs abgebaut werden (Propranolol: 1A2, 2C19, 2D6 [3, 57], Carvedilol: 1A2, 2C9, 2D6, 2E1, 3A4 $[56,58]$ und Bisoprolol: 2D6, 3A4 [59]). Es ist trotzdem kaum mit klinisch relevanten Interaktionen bei Patienten unter Therapie der HIVInfektion zu rechnen, da die Substanzen, welche über CYP metabolisiert werden, über andere Wege abgebaut werden können. Einzig Rifampicin kann zu einer signifikant erhöhten hepatischen Clearance von $\beta$-Blockern führen, was eine verminderte Wirkung zur Folge haben kann $[9,60]$.

\section{Calciumantagonisten}

Diltiazem wird über CYP3A4 abgebaut und über Pgp transportiert [61]. Verapamil wird über die CYPs 1A2, 2C8 und 3A4, Dihydropyridine nur über CYP3A4 abgebaut $[62,63]$. Verapamil und Diltiazem sind außerdem Hemmer des CYP3A4 und des Pgp [64, 65].

Interaktion zwischen Nelfinavir und Felodipin/Nifedipin. Die Gabe von Nelfinavir führte über Hemmung des CYP3A4 zu einer Erhöhung der Plasmakonzentration der Calciumantagonisten Felodipin und Nifedipin und zu einer erhöhten Toxizität [66, 67]. Ähnliche Effekte sind ebenfalls mit Verapamil und Diltiazem sowie den anderen Dihydropyridin-Calciumantagonisten zu erwarten.

Interaktion zwischen Azol-Antimykotika und Calciumantagonisten. Pharmakokinetische Studien mit Itraconazol und Felodipin wie auch mit Ketoconazol und Nisoldipin zeigen einen Anstieg der Plasmakonzentrationen der Dihydropyridin-Calciumantagonisten [68, 69]. Ähnliche Interaktionen können mit anderen Dihydropyridin-Calciumantagonisten erwartet werden $[20,68]$.

Verapamil wird über verschiedene CYPs abgebaut, weshalb der Effekt einer CYP3A4-Inhibition bei Verapamil weniger stark ausgeprägt sein könnte [20].

Interaktion zwischen Rifampicin/Rifabutin und Calciumantagonisten. Die Plasmakonzentrationen von Verapamil, Diltiazem and Dihydropyridin-Calciumantagonisten werden durch Rifampicin/Rifabutin-Gabe stark gesenkt, was zu einem Wirkungsverlust führen kann [56].
Angiotensin-II-Rezeptor-Antagonisten

Von den Angiotensin-II-Rezeptor-Antagonisten werden nur Losartan (CYP3A4 und CYP2C9) und Irbesartan (CYP2C9) über Cytochrome abgebaut [70, 71]. Das Interaktionspotential der Angiotensin-II-Rezeptor-Antagonisten ist gering. Einzig der Effekt von Losartan und Irbesartan kann verändert werden [56, 72].

Interaktion zwischen Rifampicin/Rifabutin und Losartan. Die Losartan-Clearance wird durch Rifampicin erhöht [73]. Die Dosis von Losartan muss dementsprechend erhöht werden.

Interaktion zwischen Fluconazol und Losartan. Eine pharmakokinetische Studie zeigte, dass der Metabolismus von Losartan zu seinem aktiven Metaboliten durch Fluconazol, aber nicht durch Itraconazol inhibiert wurde [74]. Die klinische Relevanz ist nicht klar, aber ein Wirkungsverlust ist möglich [74]. Ketoconazol hatte wie Itraconazol keinen Einfluss auf die Pharmakokinetik von Losartan [20].

\section{Angiotensin-Converting-Enzym-} Inhibitoren (ACEIs)

Die ACEIs (z.B. Captopril, Enalapril, Lisinopril, Perindopril, Ramipril, Quinapril, Benazepril, Cilazapril, Fosinopril, Trandolapril, Spirapril, Moexipril und Zofenopril) sind keine Substrate der Cytochrome. Sie werden renal ausgeschieden [3]. Es werden deshalb keine Interaktionen erwartet [3].

\section{Diuretika}

Diuretika werden hauptsächlich über die Nieren ausgeschieden und nicht über Cytochrome abgebaut. Aus diesem Grund sind Interaktionen mit der Therapie der HIV-Infektion unwahrscheinlich [3]. Nur Torasemid wird über CYP2C9 metabolisiert [75]. Allerdings fanden sich keine Hinweise in der Literatur auf relevante Interaktionen zu Torasemid und Therapie der HIV-Infektion.

\section{HMG-CoA-Reductase-Inhibitoren (Statine)}

Von den Statinen werden Simvastatin, Atorvastatin und Lovastatin über CYP3A4 abgebaut. Fluvastatin und Rosuvastatin werden über CYP2C9 metabolisiert, nur Pravastatin wird nicht über Cytochrome abgebaut [76]. Fluvastatin kann CYP2C19 inhibieren [77].

Interaktion zwischen HIV-Protease-Inhibitoren und Statinen. Therapie mit HIV-Protease-Inhibitoren kann zu einem Anstieg der Triglyzeride und Cholesterinkonzentration führen [78]. Auch die Therapie mit NNRTIs kann eine Dyslipid- 
ämie verstärken [78]. Die Prävalenz einer Dyslipidämie bei der Therapie mit HIV-Protease-Inhibitoren liegt ungefähr bei $75 \%$. Die Behandlung der Dyslipidämie wird durch die Eigenschaft der HIV-Protease-Inhibitoren und mancher NNRTIs, potente Inhibitoren des CYP3A4 zu sein, erheblich erschwert. Nach Koadministration von HIV-Protease-Inhibitoren und Statinen wurden Fälle von Rhabdomyolyse berichtet [79-81]. Für die Evaluation und das Management der Dyslipidämien bei HIV-infizierten Patienten unter antiretroviraler Therapie wurden in der Zwischenzeit Richtlinien publiziert [78]. Wenn eine Behandlung mit Statinen angezeigt ist, wird die Verwendung von Pravastatin oder Fluvastatin empfohlen, da sie nicht über CYP3A4 abgebaut werden [78]. Simvastatin, Lovastatin und auch Atorvastatin sollten bei diesen Patienten vermieden werden.

Interaktion zwischen Rifampicin/Rifabutin und Statinen. Rifampicin senkt die Plasmakonzentrationen der Statine, die über CYP3A4 abgebaut werden, wie z.B. Simvastatin, Atorvastatin oder Lovastatin, deutlich [9]. Es konnte ebenfalls gezeigt werden, dass Rifampicin die Plasmakonzentrationen von Pravastatin senkte (um 31\%) [82]. Da Pravastatin nicht über Cytochrome abgebaut wird, wurde spekuliert, dass andere an der Elimination beteiligte Transportproteine induziert wurden (z.B. das intestinale Multidrug-Resistenz-assoziierte Protein [MRP2]). Die Induktion dieses Transporters würde in einer verminderten enteralen Aufnahme von Pravastatin resultieren. Auch für Fluvastatin ist eine Verminderung der Plasmakonzentrationen nach Rifampicin-Gabe beobachtet worden [77].

Interaktion zwischen Azol-Antimykotika und Statinen. Azol-Antimykotika führen über eine Hemmung des CYP3A4 zu einer Verminderung des Abbaus von Simvastatin, Lovastatin und Atorvastatin [20]. Nach Kombination von Simvastatin und Itraconazol sowie Ketoconazol und Simvastatin wurde das Auftreten von Rhabdomyolysen beschrieben [83-85]. Nur einen geringen, vernachlässigbaren Effekt von Itraconazol konnte man bei gleichzeitiger Gabe mit Pravastatin beobachten [86]. Fluconazol, ein Hemmer von CYP2C9, erhöhte die AUC (,,area under the curve") von Fluvastatin, einem Substrat von CYP2C9 [87]. Fluconazol-Gabe führte aber nur zu einer marginalen Erhöhung der Rosuvastatin-Plasmakonzentrationen. Aus diesen Gründen wird bei gleichzeitiger Therapie mit Azol-Antimykotika die Therapie mit Pravastatin oder Fluvastatin, eingeschränkt in Kombination mit Fluconazol, empfohlen.

\section{Fibrate}

Bis auf Etofibrat werden die meisten Fibrate (Bezafibrat, Gemfibrozil, Clofibrat, Fenofibrat, Ciprofibrat) teilweise über das CYP3A4 abgebaut. Gemfibrozil, Clofibrat und Ciprofibrat werden zusätzlich noch glucuronidiert [88].

Es wurden keine Interaktionen zwischen antiretroviralen Medikamenten und Fibraten beschrieben [3].

\section{Schlussfolgerung}

Die Therapie von kardiovaskulär erkrankten HIV-positiven Patienten stellt eine Risikosituation dar, da aufgrund der benötigten Polypharmazie ein hohes Risiko für die Entstehung von Arzneimittelinteraktionen besteht. Durch Berücksichtigung der Informationen über die $\mathrm{Ab}$ bauwege der verwendeten Medikamente können relevante Interaktionen antizipiert und vermieden werden.

\section{Literatur}

1. Nanavati KA, Fisher SD, Miller TL, et al. HIV-related cardiovascular disease and drug interactions. Am J Cardiovasc Drugs 2004;4:315-24.

2. Greenblatt DJ, Moltke LL von, Daily JP, et al. Extensive impairment of triazolam and alprazolam clearance by short-term low-dose ritonavir: the clinical dilemma of concurrent inhibition and induction. J Clin Psychopharmacol 1999;19:293-6.

3. Fichtenbaum CJ, Gerber JG. Interactions between antiretroviral drugs and drugs used for the therapy of the metabolic complications encountered during HIV infection. Clin Pharmacokinet 2002;41:1195-211.

4. Patel IH, Zhang X, Nieforth K, et al. Pharmacokinetics, pharmacodynamics and drug interaction potential of enfuvirtide. Clin Pharmacokinet 2005;44:175-86.

5. Zhang X, Lalezari JP, Badley AD, et al. Assessment of drug-drug interaction potential of enfuvirtide in human immunodeficiency virus type 1-infected patients. Clin Pharmacol Ther 2004;75:558-68.

6. Wen X, Wang JS, Backman JT, et al. Trimethoprim and sulfamethoxazole are selective inhibitors of $\mathrm{CYP}_{2} \mathrm{C} 8$ and $\mathrm{CYP}_{2} \mathrm{C}_{9}$, respectively. Drug Metab Dispos 2002;30:631-5.

7. Trapnell CB, Jamis-Dow C, Klecker RW, et al. Metabolism of rifabutin and its 25 -desacetyl metabolite, $\mathrm{LM}_{565}$, by human liver microsomes and recombinant human cytochrome $\mathrm{P}-4503 \mathrm{~A} 4$ : relevance to clinical interaction with fluconazole. Antimicrob Agents Chemother 1997;41:924-6.

8. Jamis-Dow CA, Katki AG, Collins JM, et al. Rifampin and rifabutin and their metabolism by human liver esterases. Xenobiotica 1997;27:1015-24.

9. Niemi M, Backman JT, Fromm MF, et al. Pharmacokinetic interactions with rifampicin: clinical relevance. Clin Pharmacokinet 2003;42:819-50.

10. Reinach B, de Sousa G, Dostert P, et al. Comparative effects of rifabutin and rifampicin on cytochromes $P_{450}$ 
and UDP-glucuronosyl-transferases expression in fresh and cryopreserved human hepatocytes. Chem Biol Interact 1999;121:37-48.

11. Centers for Disease Control and Prevention. Updated guidelines for the use of rifabutin or rifampin for the treatment and prevention of tuberculosis among HIV-infected patients taking protease inhibitors or nonnucleoside reverse transcriptase inhibitors. MMWR Morb Mortal Wkly Rep 2000;49:185-9.

12. Hickman D, Sim E. N-acetyltransferase polymorphism Comparison of phenotype and genotype in humans. Biochem Pharmacol 1991;42:1007-14

13. Nishimura Y, Kurata N, Sakurai E, et al. Inhibitory effect of antituberculosis drugs on human cytochrome P450-mediated activities. J Pharmacol Sci 2004;96: 293-300.

14. Albengres E, Le Louet H, Tillement JP. Systemic antifungal agents. Drug interactions of clinical significance. Drug Saf 1998;18:83-97.

15. Pessayre D, Bentata $M$, Degott $C$, et al. Isoniazid-rifampin fulminant hepatitis. A possible consequence of the enhancement of isoniazid hepatotoxicity by enzyme induction. Gastroenterology 1977;72:284-9.

16. Steele MA, Burk RF, DesPrez RM. Toxic hepatitis with isoniazid and rifampin. A meta-analysis. Chest 1991;99:465-71.

17. Lacroix C, Hoang TP, Nouveau J, et al. Pharmacokinetics of pyrazinamide and its metabolites in healthy subjects. Eur J Clin Pharmacol 1989;36:395-400.

18. Slivka Iul, Klimniuk EV, Tabachuk OE. [Hepatotoxic effect of a combination of pyrazinamide with isoniazid and rifampicin.] Probl Tuberk 1989:39-42.

19. Breda M, Benedetti MS, Bani M, et al. Effect of rifabutin on ethambutol pharmacokinetics in healthy volunteers. Pharmacol Res 1999;40:351-6.

20. Venkatakrishnan K, Moltke LL von, Greenblatt DJ. Effects of the antifungal agents on oxidative drug metabolism: clinical relevance. Clin Pharmacokinet 2000;38:111-80.

21. Knoell KR, Young TM, Cousins ES. Potential interaction involving warfarin and ritonavir. Ann Pharmacother 1998;32:1299-302.

22. Dionisio D, Mininni S, Bartolozzi D, et al. Need for increased dose of warfarin in HIV patients taking nevirapine. AIDS 2001;15:277-8.

23. Newshan G, Tsang P. Ritonavir and warfarin interaction. AIDS 1999;13:1788-9.

24. Llibre JM, Romeu J, Lopez E, et al. Severe interaction between ritonavir and acenocoumarol. Ann Pharmacother 2002;36:621-3.

25. Cook DE, Ponte CD. Suspected trimethoprim/sulfamethoxazole-induced hypoprothrombinemia. J Fam Pract 1994:39:589-91.

26. Kaufman JM, Fauver HE Jr. Potentiation of warfarin by trimethoprim-sulfamethoxazole. Urology 1980;16: 601-3.

27. O'Reilly RA, Motley CH. Racemic warfarin and trimethoprim-sulfamethoxazole interaction in humans. Ann Intern Med 1979;91:34-6.

28. Held H. [Interaction of rifampicin with phenprocoumon (author's translation).] Dtsch Med Wochenschr 1979;104:1311-4.

29. Sennwald G. [Study of the influence of rifampin on the anticoagulant effect of acenocoumarol.] Rev Med Suisse Romande 1974;94:945-54.

30. Lee CR, Thrasher KA. Difficulties in anticoagulation management during coadministration of warfarin and rifampin. Pharmacotherapy 2001;21:1240-6.
31. Purkins L, Wood N, Kleinermans D, et al. Voriconazole potentiates warfarin-induced prothrombin time prolongation. Br J Clin Pharmacol 2003;56:Suppl 1: 24-9.

32. Self $\mathrm{TH}, \mathrm{Chrisman} \mathrm{CR}$, Baciewicz AM, et al. Isoniazid drug and food interactions. Am J Med Sci 1999;317:304-11.

33. Clarke TA, Waskell LA. The metabolism of clopidogrel is catalyzed by human cytochrome $\mathrm{P} 4503 \mathrm{~A}$ and is inhibited by atorvastatin. Drug Metab Dispos 2003; 31:53-9.

34. Ortin M, Olalla JI, Muruzabal MJ, et al. Miconazole oral gel enhances acenocoumarol anticoagulant activity: a report of three cases. Ann Pharmacother 1999;33: 175-7.

35. Richter T, Murdter TE, Heinkele G, et al. Potent mechanism-based inhibition of human CYP2B6 by clopidogrel and ticlopidine. J Pharmacol Exp Ther 2004;308: 189-97.

36. Lau WC, Waskell LA, Watkins PB, et al. Atorvastatin reduces the ability of clopidogrel to inhibit platelet aggregation: a new drug-drug interaction. Circulation 2003;107:32-7.

37. Dingemanse J, van Giersbergen PL.Clinical pharmacology of bosentan, a dual endothelin receptor antagonist. Clin Pharmacokinet 2004;43:1089-115.

38. Van Giersbergen PL, Halabi A, Dingemanse J. Singleand multiple-dose pharmacokinetics of bosentan and its interaction with ketoconazole. Br J Clin Pharmacol 2002;53:589-95.

39. Merry C, Barry MG, Ryan M, et al. Interaction of sildenafil and indinavir when co-administered to HIV-positive patients. AIDS 1999;13:F101-7.

40. Hyland R, Roe EG, Jones BC, et al. Identification of the cytochrome $\mathrm{P} 450$ enzymes involved in the $\mathrm{N}$-demethylation of sildenafil. Br J Clin Pharmacol 2001;51:239-48.

41. Muirhead GJ, Wulff MB, Fielding A, et al. Pharmacokinetic interactions between sildenafil and saquinavir/ ritonavir. Br J Clin Pharmacol 2000;50:99-107.

42. Ohyama K, Nakajima M, Nakamura S, et al. A significant role of human cytochrome $\mathrm{P}_{45} \mathrm{O}_{2} \mathrm{C} 8$ in amiodarone N-deethylation: an approach to predict the contribution with relative activity factor. Drug Metab Dispos 2000;28:1303-10.

43. Kodawara T, Masuda S, Wakasugi H, et al. Organic anion transporter oatp2-mediated interaction between digoxin and amiodarone in the rat liver. Pharm Res 2002;19:738-43.

44. Katoh $M$, Nakajima $M$, Yamazaki $H$, et al. Inhibitory effects of $\mathrm{CYP}_{3} \mathrm{~A}_{4}$ substrates and their metabolites on P-glycoprotein-mediated transport. Eur J Pharm Sci 2001;12:505-13.

45. Ohyama K, Nakajima $M$, Suzuki $M$, et al. Inhibitory effects of amiodarone and its N-deethylated metabolite on human cytochrome $\mathrm{P} 450$ activities: prediction of in vivo drug interactions. Br J Clin Pharmacol 2000;49: 244-53.

46. Lohman JJ, Reichert L, Degen LP. Antiretroviral therapy increases serum concentrations of amiodarone. Ann Pharmacother 1999;33:645-6.

47. Lea AP, Faulds D. Ritonavir. Drugs 1996;52:541-6, discussion $547-8$.

48. Zarembski DG, Fischer SA, Santucci PA, et al. Impact of rifampin on serum amiodarone concentrations in a patient with congenital heart disease. Pharmacotherapy 1999;19:249-51.

49. Sababi M, Borga O, Hultkvist-Bengtsson U. The role of P-glycoprotein in limiting intestinal regional absorption of digoxin in rats. Eur J Pharm Sci 2001;14:21-7. 
50. Ding R, Tayrouz Y, Riedel KD, et al. Substantial pharmacokinetic interaction between digoxin and ritonavir in healthy volunteers. Clin Pharmacol Ther 2004;76: 73-84.

51. Penzak SR, Shen JM, Alfaro RM, et al. Ritonavir decreas es the nonrenal clearance of digoxin in healthy volunteers with known MDR1 genotypes. Ther Drug Monit 2004;26:322-30.

52. Phillips EJ, Rachlis AR, Ito S. Digoxin toxicity and ritonavir: a drug interaction mediated through p-glycoprotein? AIDS 2003;17:1577-8.

53. Jalava KM, Partanen J, Neuvonen PJ. Itraconazole decreases renal clearance of digoxin. Ther Drug Monit 1997;19:609-13.

54. Salphati L, Benet LZ. Effects of ketoconazole on digoxin absorption and disposition in rat. Pharmacology 1998;56:308-13.

55. Greiner B, Eichelbaum M, Fritz P, et al. The role of intes tinal P-glycoprotein in the interaction of digoxin and rifampin. J Clin Invest 1999;104:147-53.

56. Flockhart DA, Tanus-Santos JE. Implications of cytochrome $\mathrm{P} 450$ interactions when prescribing medication for hypertension. Arch Intern Med 2002;162:405-12.

57. Yoshimoto K, Echizen $\mathrm{H}$, Chiba K, et al. Identification of human CYP isoforms involved in the metabolism of propranolol enantiomers - $\mathrm{N}$-desisopropylation is mediated mainly by CYP1A2. Br J Clin Pharmacol 1995;39: 421-31.

58. Oldham HG, Clarke SE. In vitro identification of the human cytochrome $\mathrm{P} 450$ enzymes involved in the metabolism of R(+)- and S(-)-carvedilol. Drug Metab Dispos 1997;25:970-7.

59. Horikiri Y, Suzuki T, Mizobe M. Pharmacokinetics and metabolism of bisoprolol enantiomers in humans. $J$ Pharm Sci 1998;87:289-94.

6o. Goldberg SV, Hanson D, Peloquin CA. Rifamycin treatment of tuberculosis in a patient receiving atenolol: less interaction with rifabutin than with rifampin. Clin Infect Dis 2003;37:607-8.

61. Pichard L, Gillet G, Fabre I, et al. Identification of the rabbit and human cytochromes P-450IIIA as the major enzymes involved in the $\mathrm{N}$-demethylation of diltiazem. Drug Metab Dispos 1990;18:711-9.

62. Tracy TS, Korzekwa KR, Gonzalez FJ, et al. Cytochrome $\mathrm{P} 450$ isoforms involved in metabolism of the enantiomers of verapamil and norverapamil. Br J Clin Pharmacol 1999;47:545-52.

63. Guengerich FP, Brian WR, Iwasaki M, et al. Oxidation of dihydropyridine calcium channel blockers and analogues by human liver cytochrome P-450 IIIA4. J Med Chem 1991;34:1838-44.

64. Zhou S, Yung Chan S, Cher Goh B, et al.Mechanism-based inhibition of cytochrome $\mathrm{P}_{450} 3 \mathrm{~A}_{4}$ by therapeutic drugs. Clin Pharmacokinet 2005;44:279-304.

65. Cornwell MM, Pastan I, Gottesman MM. Certain calcium channel blockers bind specifically to multidrug-resistant human KB carcinoma membrane vesicles and inhibit drug binding to P-glycoprotein. J Biol Chem 1987;262:2166-70.

66. Izzedine H, Launay-Vacher V, Deray G, et al. Nelfinavir and felodipine: a cytochrome P450 3A4-mediated drug interaction. Clin Pharmacol Ther 2004;75:362-3.

67. Rossi DR, Rathbun RC, Slater LN. Symptomatic orthostasis with extended-release nifedipine and protease inhibitors. Pharmacotherapy 2002;22:1312-6.

68. Jalava KM, Olkkola KT, Neuvonen PJ. Itraconazole greatly increases plasma concentrations and effects of felodipine. Clin Pharmacol Ther 1997;61:410-5.
69. Heinig R,Adelmann HG,Ahr G. The effect of ketoconazole on the pharmacokinetics, pharmacodynamics and safety of nisoldipine. Eur J Clin Pharmacol 1999;55:57-6o.

70. Stearns RA, Chakravarty PK, Chen R, et al. Biotransformation of losartan to its active carboxylic acid metabolite in human liver microsomes. Role of cytochrome $\mathrm{P} 4502 \mathrm{C}$ and $3 \mathrm{~A}$ subfamily members. Drug Metab Dispos 1995;23:207-15.

71. Marino MR, Vachharajani NN. Drug interactions with irbesartan. Clin Pharmacokinet 2001;40:605-14.

72. Unger T, Kaschina E. Drug interactions with angiotensin receptor blockers: a comparison with other antihypertensives. Drug Saf 2003;26:707-20.

73. Williamson KM, Patterson JH, McOueen RH, et al. Effects of erythromycin or rifampin on losartan pharmacokinetics in healthy volunteers. Clin Pharmacol Ther 1998;63:316-23.

74. Kaukonen KM, Olkkola KT, Neuvonen PJ. Fluconazole but not itraconazole decreases the metabolism of losartan to E-3174. Eur J Clin Pharmacol 1998;53:445-9.

75. Miners JO, Rees DL, Valente L, et al. Human hepatic cytochrome $\mathrm{P}_{450} 2 \mathrm{C} 9$ catalyzes the rate-limiting pathway of torsemide metabolism. J Pharmacol Exp Ther 1995;272:1076-81.

76. Bellosta S, Paoletti R, Corsini A. Safety of statins: focus on clinical pharmacokinetics and drug interactions. Circulation 2004;109:III50-7.

77. Scripture CD, Pieper JA. Clinical pharmacokinetics of fluvastatin. Clin Pharmacokinet 2001;40:263-81.

78. Dube MP, Stein JH, Aberg JA, et al. Guidelines for the evaluation and management of dyslipidemia in human immunodeficiency virus (HIV)-infected adults receiving antiretroviral therapy: recommendations of the HIV Medical Association of the Infectious Disease Society of America and the Adult AIDS Clinical Trials Group. Clin Infect Dis 2003;37:613-27.

79. Cheng $\mathrm{CH}$, Miller C, Lowe C, et al. Rhabdomyolysis due to probable interaction between simvastatin and ritonavir. Am J Health Syst Pharm 2002;59:728-30.

8o. Hare CB, Vu MP, Grunfeld C, et al. Simvastatin-nelfinavir interaction implicated in rhabdomyolysis and death. Clin Infect Dis 2002;35:e111-2.

81. Castro JG, Gutierrez L. Rhabdomyolysis with acute renal failure probably related to the interaction of atorvastatin and delavirdine. Am J Med 2002;112:505.

82. Kyrklund C, Backman JT, Neuvonen M, et al. Effect of rifampicin on pravastatin pharmacokinetics in healthy subjects. Br J Clin Pharmacol 2004;57:181-7.

83. Shaukat A, Benekli M, Vladutiu GD, et al. Simvastatin-fluconazole causing rhabdomyolysis. Ann Pharmacother 2003;37:1032-5.

84. Vlahakos DV, Manginas A, Chilidou D, et al. Itraconazole-induced rhabdomyolysis and acute renal failure in a heart transplant recipient treated with simvastatin and cyclosporine. Transplantation 2002;73:1962-4

85. Gilad R, Lampl Y. Rhabdomyolysis induced by simvastatin and ketoconazole treatment. Clin Neuropharmacol 1999;22:295-7.

86. Neuvonen PJ, Kantola T, Kivisto KT. Simvastatin but not pravastatin is very susceptible to interaction with the $\mathrm{CYP}_{3} \mathrm{~A}_{4}$ inhibitor itraconazole. Clin Pharmacol Ther 1998;63:332-41.

87. Kantola T, Backman JT, Niemi M, et al. Effect of fluconazole on plasma fluvastatin and pravastatin concentrations. Eur J Clin Pharmacol 2000;56:225-9.

88. Miller DB, Spence JD. Clinical pharmacokinetics of fibric acid derivatives (fibrates). Clin Pharmacokinet 1998;34:155-62. 
89. Kim AE, Dintaman JM, Waddell DS, et al. Saquinavir, an HIV protease inhibitor, is transported by P-glycoprotein. J Pharmacol Exp Ther 1998;286:1439-45.

9o. Hochman JH, Chiba M, Yamazaki M, et al. P-glycoprotein-mediated efflux of indinavir metabolites in $\mathrm{Ca}$ co- 2 cells expressing cytochrome $\mathrm{P}_{450} 3 \mathrm{~A} 4$. J Pharmacol Exp Ther 2001;298:323-30.

91. De Maat MM, Ekhart GC, Huitema AD, et al. Drug interactions between antiretroviral drugs and comedicated agents. Clin Pharmacokinet 2003;42:223-82.

92. Hsu A, Granneman GR, Bertz RJ. Ritonavir. Clinical pharmacokinetics and interactions with other anti-HIV agents. Clin Pharmacokinet 1998;35:275-91.

93. Hirani VN, Raucy JL, Lasker JM. Conversion of the HIV protease inhibitor nelfinavir to a bioactive metabolite by human liver CYP2C19. Drug Metab Dispos 2004; 32:1462-7.

94. Decker CJ, Laitinen LM, Bridson GW, et al. Metabolism of amprenavir in liver microsomes: role of $\mathrm{CYP}_{3} \mathrm{~A}_{4}$ inhibition for drug interactions. J Pharm Sci 1998;87:803-7.

95. Hurst M, Faulds D. Lopinavir. Drugs 2000;60:1371-9, discussion 1380-1.

96. Busti AJ, Hall RG, Margolis DM. Atazanavir for the treatment of human immunodeficiency virus infection. Pharmacotherapy 2004;24:1732-47.

97. Eagling VA, Back DJ, Barry MG. Differential inhibition of cytochrome $\mathrm{P} 450$ isoforms by the protease inhibitors ritonavir, saquinavir and indinavir. Br J Clin Pharmacol 1997;44:190-4.

98. Moltke LL von, Greenblatt DJ, Duan SX, et al. Inhibition of desipramine hydroxylation (cytochrome P450-2D6) in vitro by quinidine and by viral protease inhibitors: relation to drug interactions in vivo. J Pharm Sci 1998;87:1184-9.

99. Washington CB, Duran GE, Man MC, et al. Interaction of anti-HIV protease inhibitors with the multidrug transporter P-glycoprotein (P-gp) in human cultured cells. J Acquir Immune Defic Syndr Hum Retrovirol 1998;19:203-9.

100. Hesse LM, Moltke LL von, Shader RI, et al. Ritonavir, efavirenz, and nelfinavir inhibit CYP2B6 activity in vitro: potential drug interactions with bupropion. Drug Metab Dispos 2001;29:100-2.

101. Moltke LL von, Greenblatt DJ, Grassi JM, et al. Protease inhibitors as inhibitors of human cytochromes P450: high risk associated with ritonavir. J Clin Pharmacol 1998;38:106-11.

102. Gutmann H, Fricker $G$, Drewe J, et al. Interactions of HIV protease inhibitors with ATP-dependent drug export proteins. Mol Pharmacol 1999;56:383-9.

103. Lillibridge $\mathrm{JH}$, Liang $\mathrm{BH}$, Kerr BM, et al. Characterization of the selectivity and mechanism of human cytochrome $\mathrm{P} 450$ inhibition by the human immunodeficiency virus-protease inhibitor nelfinavir mesylate. Drug Metab Dispos 1998;26:6o9-16.

104. Vishnuvardhan D, Moltke LL, Richert C, et al. Lopinavir: acute exposure inhibits P-glycoprotein; extended exposure induces P-glycoprotein. AIDS 2003;17:1092-4.

105. Kageyama $M$, Namiki $H$, Fukushima $H$, et al. Effect of chronic administration of ritonavir on function of cytochrome $\mathrm{P}_{450} 3 \mathrm{~A}$ and $\mathrm{P}$-glycoprotein in rats. Biol Pharm Bull 2005;28:130-7.
106. Frye RF, Bertz RJ, Granneman GR, et al. Effect of ritonavir on CYP1A2, 2C19, and 2E1 activities in vivo. Clin Pharmacol Ther 1998;63:148.

107. Huang L, Wring SA, Woolley JL, et al. Induction of P-glycoprotein and cytochrome $\mathrm{P} 4503 \mathrm{~A}$ by HIV protease inhibitors. Drug Metab Dispos 2001;29:754-60.

108. PerloffES, Duan SX, Skolnik PR, et al. Atazanavir: effects on P-glycoprotein transport and $\mathrm{CYP}_{3} \mathrm{~A}$ metabolism in vitro. Drug Metab Dispos 2005;33:764-70.

109. Smith PF, DiCenzo R, Morse GD. Clinical pharmacokinetics of non-nucleoside reverse transcriptase inhibitors. Clin Pharmacokinet 2001;40:893-905.

110. Erickson DA, Mather G, Trager WF, et al. Characterization of the in vitro biotransformation of the HIV-1 reverse transcriptase inhibitor nevirapine by human hepatic cytochromes P-450. Drug Metab Dispos 1999; 27:1488-95.

111. Voorman RL, Payne NA, Wienkers LC, et al. Interaction of delavirdine with human liver microsomal cytochrome $\mathrm{P}_{450}$ : inhibition of $\mathrm{CYP}_{2} \mathrm{C}_{9}, \mathrm{CYP}_{2} \mathrm{C}_{19}$, and CYP2D6. Drug Metab Dispos 2001;29:41-7.

112. Ward BA, Gorski JC, Jones DR, et al. The cytochrome $\mathrm{P}_{450}$ 2B6 (CYP2B6) is the main catalyst of efavirenz primary and secondary metabolism: implication for HIV/ AIDS therapy and utility of efavirenz as a substrate marker of CYP2B6 catalytic activity. J Pharmacol Exp Ther 2003;306:287-300.

113. Moltke LL von, Greenblatt DJ, Granda BW, et al. Inhibition of human cytochrome $\mathrm{P} 450$ isoforms by nonnucleoside reverse transcriptase inhibitors. J Clin Pharmacol 2001;41:85-91.

114. Stormer E, Moltke LL von, Perloff MD, et al. Differential modulation of P-glycoprotein expression and activity by non-nucleoside HIV-1 reverse transcriptase inhibitors in cell culture. Pharm Res 2002;19:1038-45.

115. Mouly S, Lown KS, Kornhauser D, et al. Hepatic but not intestinal $\mathrm{CYP}_{3} \mathrm{~A}_{4}$ displays dose-dependent induction by efavirenz in humans. Clin Pharmacol Ther 2002;72:1-9.

116. Jeu L, Piacenti FJ, Lyakhovetskiy AG, et al. Voriconazole. Clin Ther 2003;25:1321-81.

117. Buchkowsky SS, Partovi N, Ensom MH. Clinical pharmacokinetic monitoring of itraconazole is warranted in only a subset of patients. Ther Drug Monit 2005;27:322-33.

118. Daneshmend TK, Warnock DW. Clinical pharmacokinetics of ketoconazole. Clin Pharmacokinet 1988;14:13-34.

119. Ufer M, Svensson JO, Krausz KW, et al. Identification of cytochromes $\mathrm{P}_{45} \mathrm{O}_{2} \mathrm{C} 9$ and $3 \mathrm{~A} 4$ as the major catalysts of phenprocoumon hydroxylation in vitro. Eur J Clin Pharmacol 2004;60:173-82.

120. Thijssen $\mathrm{HH}$, Flinois JP, Beaune $\mathrm{PH}$. Cytochrome $\mathrm{P} 45 \mathrm{O} 2 \mathrm{C} 9$ is the principal catalyst of racemic acenocoumarol hydroxylation reactions in human liver microsomes. Drug Metab Dispos 2000;28:1284-90.

121. Rettie AE, Korzekwa KR, Kunze KL, et al. Hydroxylation of warfarin by human CDNA-expressed cytochrome P-450: a role for P-4502C9 in the etiology of (S)-warfarin-drug interactions. Chem Res Toxicol 1992;5:54-9.

122. Zhang Z, Fasco MJ, Huang Z, et al. Human cytochromes P4501A1 and P4501A2: R-warfarin metabolism as a probe. Drug Metab Dispos 1995;23:1339-46.

\section{Korrespondenzanschrift}

Prof. Dr. Jürgen Drewe Abteilung für Klinische Pharmakologie \& Toxikologie Universitätsspital Basel Hebelstraße 2 4031 Basel Schweiz Telefon (+41/61) 265-3848, Fax -8581 E-Mail:juergen.drewe@ unibas.ch 